\title{
Germanica
}

\section{L'histoire comme farce ou la déconstruction de l'histoire dans Turlupinde Leo Perutz}

Die Historie als Posse oder die Dekonstruktion der Historie in Turlupin von Leo Perutz

Jean-Pierre Chassagne

\section{OpenEdition \\ Journals}

Édition électronique

URL : http://journals.openedition.org/germanica/1276

DOI : $10.4000 /$ germanica. 1276

ISSN : 2107-0784

\section{Éditeur}

Université de Lille

\section{Édition imprimée}

Date de publication : 1 décembre 1998

Pagination : 143-161

ISBN : 0984-2632F

ISSN : 0984-2632

\section{Référence électronique}

Jean-Pierre Chassagne, «L'histoire comme farce ou la déconstruction de l'histoire dans Turlupinde Leo Perutz », Germanica [En ligne], 23 | 1998, mis en ligne le 27 janvier 2012, consulté le 06 octobre 2020 URL : http://journals.openedition.org/germanica/1276 ; DOI : https://doi.org/10.4000/germanica.1276

Ce document a été généré automatiquement le 6 octobre 2020.

(C) Tous droits réservés 


\section{L'histoire comme farce ou la déconstruction de l'histoire dans Turlupin ${ }^{1}$ de Leo Perutz}

Die Historie als Posse oder die Dekonstruktion der Historie in Turlupin von Leo Perutz

Jean-Pierre Chassagne

1 Ce n'est pas chose aisée que de dégager la spécificité du roman historique pérutzien par rapport aux modèles du genre antérieurs et contemporains car il ne se laisse pas appréhender à travers les critères habituellement retenus pour l'analyse de cette tradition littéraire. En effet, Perutz n'envisage l'histoire ni comme la préhistoire du présent, ${ }^{2}$ ni comme un refuge contre une actualité décevante, pas plus qu'il ne l'investit d'une dimension messianique. Précédant A. Döblin selon lequel le roman historique, c'est d'abord du roman, et deuxièmement ce n'est pas de l'histoire $e^{3}$,il entretient avec le passé historique une relation extrêmement irrévérencieuse et distanciée. Ce faisant, il délimite le territoire spécifique de la littérature comme un espace de jeu avec des signifiants multiples. Ce jeu questionne l'histoire, tout en refusant de lui donner un sens univoque. Le roman Turlupinillustre de façon exemplaire le traitement ludique de l'histoire. Par ailleurs, il manie l'ironie comme un outil de déconstruction visant aussi bien les pratiques prétendument infaillibles de l'historiographie que l'interprétation téléologique et rationaliste de 1'historicisme.

\section{La parodie de l'enquête historiographique}

2 L'action du roman est située en France sous le règne de Louis XIII. L'intrigue évoque le conflit qui oppose le cardinal de Richelieu à la noblesse française prête à tout pour sauvegarder ses privilèges menacés. La fable du roman débute par l'annonce d'un événement qui doit survenir le 11 novembre 1642 et bouleversera profondément le visage de la France. Richelieu projette en effet le massacre de l'aristocratie afin de 
doter son pays d'une république. Or, comme chacun sait, la Révolution française n'aura lieu qu'en 1789. Aussi la tâche du romancier consiste-t-elle à nous expliquer comment un simple perruquier parisien nommé Turlupin a pu retarder de 147 ans le cours de l'histoire.

3 Par le choix du sujet étranger à l'univers de référence germanique, Perutz se démarque de nombre de ses prédécesseurs ou de ses contemporains qui puisaient le plus souvent dans le passé national la matière susceptible d'illustrer leur vision conservatrice ou progressiste du monde. On pourrait même considérer le recours à l'Histoire de France, et la date du 11 novembre, cinq ans après la défaite de 1918, comme une forme de provocation, atténuée il est vrai, par le ton adopté qui est celui de la comédie burlesque.

4 Dans le premier chapitre, la dérision préside à l'exposition des sources historiographiques. Tel un historien consciencieux et qualifié, le narrateur mène son récit sur le mode de l'enquête qu'il étaye par de multiples documents de diverses origines. Il feint ainsi d'éclairer les événements du 11 novembre 1642 qui ont, dit-il, agité la capitale française. La première source citée relate une anecdote qui constitue le point d'entrée dans l'histoire. Le récit s'ouvre par un incident paradoxal : le formidable éclat de rire d'un condamné, ancien greffier de tribunal, auquel on vient d'annoncer la lourde sentence de onze ans de galère et de six cent livres d'amende. Curieusement, ce procès ne constitue que l'entrée en matière : le condamné Michel Babaut disparaît de la diégèse ${ }^{4}$ aussi vite qu'il était apparu. Son cas n'est en fait qu'un prétexte pour installer le référent pseudo-historique et introduire la date du 11 novembre 1642 qui constitue le pivot de toute l'intrigue. Néanmoins le mystère reste entier sur les raisons de la désinvolture du condamné. Suit l'évocation de la rumeur publique qui, par anticipation, prête à cette date-clé une signification inconnue, ce qui ne fait qu'accroître le suspense en installant le lecteur dans l'attente de la révélation des faits indispensable à la compréhension de l'énigme.

5 Le récit affirme avec insistance sa prétention à l'exactitude et à la rigueur scientifique en citant des sources dont la diversité et la complémentarité ne sont pas sans rappeler, du moins en apparence, le travail rigoureux de l'archiviste historiographe soucieux d'atteindre à l'objectivité par la juxtaposition de différents points de vue. Les documents cités sont de triple nature : certains ont une fonction proleptique ${ }^{5}$, d'autres sont contemporains de l'événement, toujours inconnu, auquel ils sont immédiatement liés, les derniers sont de nature analeptique ${ }^{6}$ et retracent les différentes étapes de la réception du 11 novembre 1642 à des époques ultérieures.

6 Cette apparente exhaustivité instaure une complicité entre le lecteur et le narrateur. En effet, en citant des documents d'archives, ce dernier feint de divulguer la parole officielle et la mémoire d'un peuple habituellement inaccessibles au grand public. Par ailleurs, ces sources épuisent toutes les pistes possibles que peut suivre un chercheur. A un premier niveau de lecture, elles installent le lecteur dans l'illusion que toute la vérité va lui être révélée sur les faits survenus le 11 novembre 1642. Aussi le référent de fiction se donne à lire comme un référent historique. L'importance exceptionnelle de cette date est attestée tour à tour par un pamphlet, un document précieusement conservé aux Archives Nationales, les mémoires d'un contemporain, enfin par des extraits de presse de l'époque. Cependant l'accumulation des sources, la surenchère d'informations et le zèle avec lequel le narrateur suggère la scientificité de sa démarche finissent par alerter le lecteur attentif. Les soupçons de celui-ci s'avèrent justifiés 
lorsqu'il découvre le contenu on ne peut plus sibyllin de ces sources. Loin de le renseigner sur la nature des faits qui auraient dû transformer le visage de la France le jour de la Saint-Martin (à la date du calendrier, le narrateur substitue discrètement le saint patron plus pittoresque et moins officiel) les témoignages cités renforcent sa perplexité et sa curiosité, dans la mesure où ils ne font que reprendre la rumeur publique qui incita la population à placer tous ses espoirs dans le Jeu de volant du 11 novembre 1642. La juxtaposition de la parole populaire, constituée de rumeurs incertaines et anonymes, et de témoignages de personnages illustres est un pied de nez à l'historiographie des sources.

7 Le narrateur joue par ailleurs avec la méfiance, et la curiosité du lecteur, en restant muet sur les faits qui vont bouleverser le cours de l'histoire lors du Jeu de volant auquel un certain monsieur de Saint- Chéron a convoqué ses amis. Les "informations" contenues dans les sources dissimulent plus qu'elles ne révèlent la signification capitale de cet événement. L'amateur de roman historique ne peut alors qu'être dérouté par une telle entrée en matière qui se montre plus soucieuse de provoquer le rire et de créer le suspense que d'installer le lecteur dans l'atmosphère d'une époque. La rétention de l'information essentielle à la compréhension procède de la déconstruction dans la mesure où elle diffère le sens en un point ultérieur au présent du texte ${ }^{7}$. Le lecteur se voit donc privé d'un savoir partagé par tous les personnages de la diégèse, ce qui ne peut qu'augmenter son impatience d'apprendre enfin la vérité. Or, un texte répertorié aux Archives Nationales ne s'avère pas plus instructif qu'un pamphlet signé Etienne qui dit tout, mais qui ne fait que confirmer ce que les Parisiens savaient déjà ${ }^{8}$ :

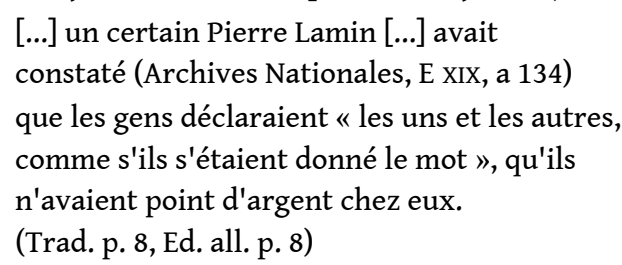

8 Dans cet exemple, la voix du narrateur relègue au second plan celle du témoin, ce qui illustre la dilution du sens lors de son colportage et de son filtrage par différentes sources d'information. Il faut par ailleurs noter l'effet hautement comique du passage cité entre guillemets, alors que sa teneur en information est nulle. Suit le récit d'une aristocrate, madame d'Ouchy, qui relate le départ précipité de sa famille résignée à fuir la capitale le 11 novembre 1642. D'une part, ce témoignage n'apporte rien de nouveau, puisqu'il émane d'une personne ignorant les faits qui ont provoqué ce départ, d'autre part il ne constitue qu'une mince anecdote, elle-même en partie abrégée et résumée par l'éditeur de ces mémoires rédigés vingt ans après les événements relatés. En outre, ce texte fut publié, nous dit-on, en 1892, soit deux cent trente ans après sa rédaction. Ce double décalage temporel est de toute évidence une mise en cause du travail des historiens dont les méthodes d'investigation sont tournées en dérision. Non seulement le narrateur souligne le caractère anecdotique de ce témoignage, qui est d'autant plus sujet à caution que les " papiers personnels » de madame d'Ouchy ont fait l'objet d'une sélection par leur éditeur, et ne constituent donc pas une source directe et intégrale, mais il se rit aussi de la vulgarisation de l'histoire au XIX ${ }^{\mathrm{e}}$ siècle : l'engouement du public, et des auteurs, pour l'autobiographie ou la petite histoire anecdotique sont ainsi tournés en dérision. 
9 L'accumulation incongrue de toutes ces références se lit a posteriori comme la chronique d'un non-événement annoncé à grand renfort de moyens, mais dont on ignore toujours la nature.

[...] car la Saint-Martin était passée et

rien ne s'était produit (Ibid., p. 9, Ed. all.

p. 7)

10 La révélation différée des faits aboutit donc à une vacuité du sens. Le lecteur est confronté à un récit, qui s'autodétruit dans la mesure où il annonce un non-événement. Mais sa curiosité est paradoxalement maintenue en éveil par ce qui s'avère être un nonrécit et il brûle de savoir pourquoi les événements annoncés, quels qu'ils soient, n'ont pas eu lieu.

11 Le narrateur présente ensuite une nouvelle série de sources qui atteste du nonévénement tel qu'il fut enregistré par ses contemporains. Une fois encore, la prétention à l'objectivité est simulée par la juxtaposition de coupures de presse destinées aux deux classes antagonistes de la société française d'alors, à savoir la Gazette de la courpour la noblesse et la Gazette de Francepour le Tiers-Etat. Or, si l'on se réfère aux faits rapportés dans les chapitres suivants du roman, on s'aperçoit qu'aucun des deux journaux ne permet à ses lecteurs de comprendre le sens des événements évoqués, lesquels sont traités comme de simples faits divers. La Gazette de la cour mentionne bien une émeute aux abords de l'hôtel Lavan, mais ne dit mot sur les deux victimes, ni sur l'enjeu de ce soulèvement populaire. En outre, elle met l'incident sur le même plan qu'un duel entre deux aristocrates, chose fort courante à l'époque. Ce faisant, elle le banalise. Quant à la Gazette de France, elle satisfait le goût du roturier pour la sensation en relatant des faits divers, mais elle ne fait aucune allusion au soulèvement avorté du peuple de Paris dont elle veut, de toute évidence, ménager la susceptibilité, à moins que la censure ne lui ait imposé le silence. Le narrateur pousse la dérision jusqu'à relativiser l'intérêt des sources citées qui n'ont pas rempli leur fonction et ne rapportent que des incidents mineurs qui faisaient partie, dans le Paris de Richelieu et de Louis XIII, des choses quotidiennes(p. 9, Ed. all. p. 7).

12 L'historiographe amateur ne s'avoue pas pour autant vaincu et va, dans un troisième mouvement, orienter sa recherche vers la réception du non-événement aux époques qui lui ont succédé. C'est la mémoire populaire qui va garantir une longue vie au souvenir de la Saint-Martin 1642 et assurer son entrée dans la légende grâce aux chansons de rue, aux feuilles imprimées, ou aux impromptus de comédiens ambulants. Le narrateur note, non sans ironie, les mutations de sens subies au fil du temps par cette date, qui devint au XVIII ${ }^{\mathrm{e}}$ siècle la Saint-Glinglin,jusqu'à sa dernière évocation dans les Mémoires, correspondance et ouvrages inédits, Paris, 1830de Diderot. La référence à ce nom illustre est de nature à légitimer la curiosité du lecteur qui sera cependant, une fois de plus, abusé par un narrateur plus enclin à brouiller les pistes qu'à se livrer à une analyse scientifique de la source proposée. L'âge du philosophe permet de déduire que le document cité date de 1738 , c'est-à-dire d'une époque à laquelle Diderot n'avait encore rien publié! Pour un lecteur non-spécialiste, cette date n'a certes rien de choquant et renforce l'effet de vraisemblance. Le lecteur cultivé peut-il conclure à la négligence de l'auteur? Non, car Perutz fait preuve ici d'une désinvolture affichée en suggérant une date dont il sait qu'elle s'effondrera après vérification. Donc, ce référent historique se donne a posteriorià relire comme un piège à retardement, comme une source inauthentique historiquement fausse. Il s'agit du commentaire qu'inspira à 
Diderot l'exécution barbare de l'assassin Saulnier (p.10, Ed.all. p. 8) et dans lequel l'évocation de la Saint-Martin prend une dimension prophétique: cette date, que l'écrivain projette dans le futur, devient en effet pour lui symbole de liberté d'expression et de justice, ce qui autorise le lecteur à voir dans les réflexions du philosophe des Lumières une allusion à la Révolution Française de 1789 :

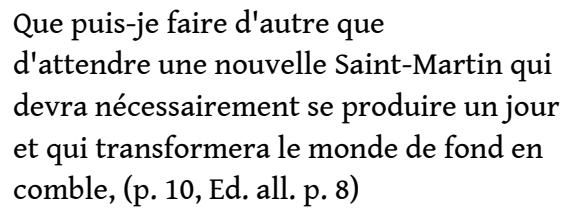

Tout en remettant en cause, comme nous le verrons plus tard, la pertinence d'une telle mise en perspective de l'histoire, le narrateur illustre la dissémination du sens d'un signe, ici la date du 11 novembre 1642, à travers sa réinterprétation à différentes époques. Ce que Diderot déplore, face à la barbarie de son temps, c'est justement l'avènement différé de ce que représente pour lui la Saint-Martin. Le sens dont il investit cette date renvoie à la fois au passé, dont il tire sa substance, et à un avenir meilleur censé l'enrichir d'une nouvelle charge sémantique. Le lecteur, pour sa part, reste totalement étranger à ces spéculations. Elles demeurent sibyllines pour lui dans la mesure où il ne connaît ni les acteurs, ni les faits auquel renvoie sans arrêt le récit. Il n'en reste pas moins que la référence d'un précurseur illustre de la Révolution française à ce qui est déjà identifié comme un non-événement relève de la dérision.

Puis arrive l'instant tant attendu par le lecteur où la signification du 11 novembre 1642 va lui être enfin révélée. Le narrateur se plaît cette fois à évoquer en passant, et sans les citer textuellement, des sources sérieuses ou des travaux scientifiques dont il souligne la banalité alors qu'ils sont les seuls à faire toute la lumière sur le complot de Richelieu contre la noblesse de France :

$$
\begin{aligned}
& \text { Des documents qui dormaient depuis } \\
& \text { des siècles dans l'obscurité des Archives } \\
& \text { ont été mis à jour [...]. Il n'est pas } \\
& \text { nécessaire de renvoyer aux études de } \\
& \text { d'Avenel, de R. Perkins et de D. Rocas. } \\
& \text { (pp. 10-11, Ed. all. p. } 8 \text { ) }
\end{aligned}
$$

Le narrateur se distancie donc avec ostentation de l'historiographie officielle dont les travaux ont consisté, selon lui, à démontrer une évidence. Ce faisant, il précise les limites de la fiction historique qui se situe à l'écart de l'activité scientifique et n'est pas soumise à l'obligation de vérité. En nommant enfin le cardinal de Richelieu comme le commanditaire de la révolution avortée, le récit laisse entendre à son décrypteur que l'information tant attendue va enfin lui être révélée. Cependant la déconstruction poursuit son œuvre en différant à nouveau le sens dans un ailleurs du texte. Le narrateur reprend le jeu subversif de l'énumération des sources fictives. Celles-ci sont censées confirmer la thèse du projet sanguinaire du cardinal puisqu'elles émanent des proches de l'ecclésiastique qui ont été initiés au «secret des Dieux». Or, comme précédemment, le lecteur est frustré car les "témoins", muselés par l'obligation de discrétion, ou anéantis par la cruauté d'un tel complot, se contentent d'allusions évasives et laconiques qui sont cependant citées littéralement. En vérité, ces sources masquent plus qu'elles ne dévoilent. Elles nimbent le projet de Richelieu d'un halo d'irréalité et de légende qui est aussi celui de la fiction littéraire en gestation. Après avoir simulé l'enquête dans toutes les couches sociales, puis à différentes époques, le récit feint de toucher finalement au cœur de la vérité en recueillant l'information à la 
source première, à savoir sous la plume du cardinal. Et cette parole ne saurait d'autant moins être mise en doute qu'elle est inscrite dans la bible du prélat :

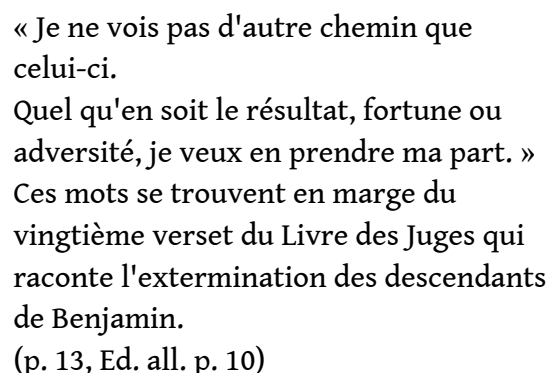

Or, l'exégèse d'une annotation aussi évasive que celle-ci, par le recours à un passage de la Sainte Ecriture, ne va pas sans poser quelque problème. Il est en effet malaisé de déterminer après coup quel texte éclaire l'autre, d'autant plus qu'une annotation manuscrite est habituellement le commentaire du texte imprimé auquel elle est juxtaposée, et non l'inverse. Le mystère reste donc entier, ce qui n'empêche pas le narrateur de réitérer son interprétation du non-événement de la Saint-Martin comme l'échec de la première tentative de révolution en France. Le repérage du sens à partir des divers documents insignifiants dévoile l'intention parodique du roman qui se rit de la pratique hypertextuelle de l'historiographie. L'accumulation de sources, dont chacune illustre un point de vue différent et subjectif sur un même fait, ne contribue pas à faire toute la lumière sur les événements évoqués. Elle matérialise la dissémination du sens. Par ailleurs, celui-ci est constamment différé d'un moment à l'autre de l'histoire de France, ainsi que vers un avenir du texte de façon à suggérer au lecteur que le message du roman et la vérité sur le 11 novembre 1642 vont lui être révélées par l'intrigue qui se noue au chapitre deux.

\section{L'opacification de l'histoire}

L'exposition du plan de Richelieu en fin de chapitre 1 permet de faire entrer en scène les actants de l'intrigue et de préciser le rôle de la fiction par rapport à l'histoire. Il est clair que Perutz congédie d'entrée de jeu l'histoire telle qu'elle est relatée par l'historiographie. Le projet paradoxal du cardinal, qui nous est présenté ici, se nourrit certes du conflit permanent, attesté par les textes, entre l'homme politique et la noblesse française de l'époque, mais Perutz s'autorise quelque liberté par rapport aux faits : transformer en champion du républicanisme celui qui a lutté toute sa vie pour renforcer la monarchie absolue a, en effet, de quoi heurter les esprits rationalistes épris de vérité historique. Celle-ci importe peu pour Perutz qui la remet en question en greffant la fiction sur les zones d'ombre de l'histoire :

C'est un des grands mystères de l'évolutionde l'humanité que la

Révolution française n'ait éclaté qu'en

1789.

(p. 13, Ed. all. p. 10, souligné par nous

J.-P. C.)

18 L'écrivain délimite son territoire en marge de l'histoire officielle. En rapprochant différentes époques et différents faits qui n'ont apparemment rien en commun, il précise sa conception $\mathrm{du}$ roman historique: celui-ci devient un terrain 
d'expérimentation avec les données de l'histoire, un jeu de déconstruction des signifiés habituellement projetés sur celle-ci.

L'annonce du dénouement de l'intrigue en début de roman s'inscrit dans cette stratégie qui sape les fondements de toutes les certitudes: le mystérieux échec des idées révolutionnaires de Richelieu remet en question la conception téléologique de l'histoire comme processus évolutif, et transforme cette dernière en énigme que seul le roman peut résoudre en nous révélant les causes du non-événement. On peut donc enregistrer un déplacement de l'intérêt du lecteur programmé par le texte : il ne s'agit plus de savoir, comme dans un livre d'histoire, ce qui s'est produit, mais de comprendre pourquoi l'histoire ne s'est pas déroulée autrement qu'elle ne l'a fait. Perutz s'installe donc dans un carrefour narratif de son récit ${ }^{9}$. Celui-ci ne se donne plus à lire comme une reconstitution historique, mais comme une fiction qui s'épanouit dans un repli de l'histoire.

Dès le deuxième chapitre, l'anecdote supplante la "Grande Histoire » et passe au premier plan. Le personnage fictif de Turlupin, un modeste barbier, occupera le devant de la scène jusqu'à la conclusion du récit. Fait d'autant plus significatif que ce bouffon de comédie, que rien ne prédestinait à entrer dans l'histoire, va infléchir le destin de la France, sans le savoir. Comme toujours chez Perutz, l'histoire échappe à ses prétendus acteurs, c'est à dire à ses " héros ", et s'accomplit en marge du pouvoir. Ce sont donc les petits faits, comme l'ambition sociale d'un pauvre roturier, et ses efforts désespérés pour se faire admettre dans le grand monde, qui parasitent l'histoire pour alimenter la fiction. Il n'est donc pas étonnant que le roman se termine par l'évocation de petites gens, comme le voleur Jacques Maugeret, et la veuve Sabot dont la vie quotidienne renvoie à celle qu'aurait pu mener Turlupin, et n'a en rien été modifiée par l'avortement de la révolution. L'échec des projets du titan Richelieun'est que l'écume des choses. Néanmoins les aventures du barbier ne sont pas de nature à donner un sens à ce chapitre trouble de l'histoire.

21 Plus le lecteur avance dans le récit focalisé sur Turlupin, plus il est confronté à des énigmes, car son degré de savoir et de compréhension des faits n'excède pas celui du personnage principal à l'esprit borné qui est aveuglé par sa subjectivité. La reconstitution de la vérité n'est donc pas secondée par une instance de narration auctoriale qui pourrait confirmer ou infirmer la version des faits de Turlupin. Aussi le lecteur tâtonne de question en question : de qui célèbre-t-on les obsèques dans l'église où Turlupin s'est rendu par erreur? Pourquoi madame de Lavan fixe-t-elle le barbier avec insistance? Est-elle vraiment sa mère? Turlupin parviendra-t-il à se faire reconnaître comme son fils illégitime? Tout comme le premier chapitre l'avait laissé entendre, le "roman d'histoire» se mue en roman à suspense dont les péripéties maintiennent la curiosité du lecteur en éveil. Cette irrévérence à l'égard du roman historique classique ne fait pas pour autant du texte pérutzien un pur produit de la Trivialliteratur. Les énigmes et le suspense sont mis au service de la déconstruction. Cette dernière remet en question les théories préconçues qui président à l'interprétation de l'histoire.

22 Loin de renforcer les certitudes, le roman pérutzien cultive l'ambiguïté et joue sur la confusion et le malentendu. Turlupin commet sa première erreur lorsqu'il se méprend sur l'identité de celui dont on célèbre les obsèques en l'église des Trinitaires. Alors qu'il est persuadé d'assister aux funérailles d'un mendiant auquel il n'a pas fait l'aumône avant sa mort, il croit identifier en la duchesse de Lavan, l'épouse du défunt, sa mère 
qui le regarde fixement parce que, s'imagine-t-il, elle reconnaît en lui le fils illégitime qu'elle aurait abandonné à sa naissance. Ce malentendu sera à l'origine de tous les événements ultérieurs. Aussi peut-on dire que la Révolution française a été retardée de cent quarante sept ans parce que Turlupin s'est mépris sur le nom d'un défunt dont on célébrait les funérailles. Par ailleurs, l'identité des actants de l'intrigue est sujette à des fluctuations: parce qu'il refuse ses basses origines, Turlupin se persuade qu'il est l'enfant illégitime d'une aristocrate. Pour parvenir à ses fins et s'introduire dans l'hôtel de Lavan, il usurpe l'identité d'un gentilhomme dont on vient de lui apprendre la mort. Quant à Saint-Chéron, le chef de la rébellion qui défend les intérêts de Richelieu, il se fait lui-aussi passer pour un autre et fréquente le salon du barbier sous le nom de monsieur Gaspard. La tranche d'histoire relatée dans ce roman est donc présentée comme une mascarade: chacun se dissimule à son adversaire et ne l'identifie pas clairement, de telle sorte que les enjeux de l'affrontement des deux camps adverses ne sont pas nettement repérables ni repérés.

L'antihéros Turlupin incarne à ses dépens l'aveuglement qui préside à la marche de l'histoire. Contrairement au personnage du roman réaliste, il ne possède aucun des attributs du héros moyen (mittlerer Held)défini par Lukács ${ }^{10}$. Le barbier n'est en effet ni un type, ni un résumé d'histoire. C'est un être sans identité stable qui traverse les événements sans les comprendre, puis meurt dans l'anonymat. Il ne représente aucun groupe social. Il n'agit qu'au nom de ses intérêts personnels qui sont contraires à ceux de sa classe. Le clivage de son moi le rend prisonnier de ses rêves de gloire et substitue à la réalité un monde chimérique dans lequel il croit accéder à la reconnaissance sociale en levant tous les obstacles qui le séparent de l'aristocratie. Cependant le double programme narratif ${ }^{11}$ dans lequel il est engagé condamne son agir à l'échec. L'action conjonctive de Turlupin (assurer la victoire au camp révolutionnaire qui représente les intérêts de sa classe) est contrecarrée par sa motivation personnelle (être reconnu comme aristocrate de naissance). Le passage d'un programme narratif à un autre entraîne une inversion de l'Adjuvant et de l'opposant sur l'axe secondaire du pouvoir : sur le plan des intérêts individuels, le barbier est secondé par la noblesse (son Adjuvant) et menacé par Saint-Chéron (son Opposant) qui sert la cause de la plèbe. Sur le plan politique, Turlupin, qui se fait passer pour monsieur de Josselin, devient l'allié de Saint-Chéron pour espionner les aristocrates dans l'hôtel de Lavan. Donc, le perruquier pourrait choisir entre la rébellion, dont le triomphe rendrait caduque son ambition sociale, ou la contre-révolution au prix d'une trahison des intérêts de sa classe. Or, l'ironie du récit réside dans le fait que Turlupin ne fait aucun choix lucide dans la mesure où ses actes ne sont pas dictés par une conscience politique, mais par l'opportunité du moment. L'enjeu de l'histoire n'est donc pas de nature idéologique, et ce n'est pas la quête d'un sens qui préside au devenir historique. Perdant quelle que soit l'issue de l'affrontement des forces en présence, Turlupin se condamne à l'échec, il meurt pour rien comme il a agi pour rien : la duchesse de Lavan, qui est aveugle depuis son enfance, ne l'identifie pas comme son fils. Cependant sa cécité ne l'empêche pas de voir la vérité avec ses mains: celles-ci détectent, en explorant les traits du défunt Turlupin, un visage bien commun (p. 180, Ed. all. p. 149). qui ne peut être celui d'un gentilhomme. Un voyant aveuglé, une aveugle voyante, tels sont les ambiguïtés et les paradoxes avec lesquels s'écrit l'histoire, mais qu'aucune dialectique ne permet de dépasser. 


\section{L'histoire comme turlupinade}

elle-même avec, comme point aveugle, un non-événement. Le piétinement de l'histoire est ainsi suggéré. D'autre part, affirmer que la Révolution française aurait pu avoir lieu 147 ans avant la date connue, car toutes les conditions étaient réunies pour cela, revient à dire que le cours des événements est livré au hasard. Autrement dit, la Révolution française aurait tout aussi bien pu ne pas avoir lieu en 1789. Mais le travail de sape orchestré par Perutz est encore plus subversif qu'il n'y paraît à la première lecture. Par le jeu des comparaisons et de la dissémination du sens, deux épisodes, que les historiens ont coutume de situer aux antipodes l'un de l'autre, à savoir la SaintBarthélémy et la Révolution française, se voient juxtaposés et évalués, voire dévalués par ce rapprochement. L'écriture de Perutz suggère des parallèles, fait circuler et évoluer le sens d'une époque à l'autre, elle crée de nouveaux réseaux de signification afin de mettre en évidence l'ambivalence de tout processus historique.

L'évocation du représentant de la pensée rationaliste qu'est Diderot relève du même esprit : l'idéalisme naïf du philosophe des Lumières est mis à la dure épreuve de la réalité :

Si Diderot avait pu se douter du mystère effrayant que cachait cette tournure, il n'eût certainement pas écrit ces mots, lui qui rêvait d'un renouveau sans effusion de sang, d'une révolution des esprits, (p. 10, Ed. all. p. 8)

Cette phrase est une mise en abyme ${ }^{12}$ de tout le roman: Diderot évoque le renouveau auquel il aspire sans se douter que celui-ci ne se manifestera pas sous la forme souhaitée. De la même façon, le récit qui annonce un non-événement se clôt sur luimême et se prive de tout avenir. Perutz se sert du représentant d'un optimisme et 
d'une foi dans le progrès, qu'il ne partage pas, pour dénoncer les violences de la Révolution française. Il met en évidence l'opacité de la langue qui se traduit par l'ébranlement de l'identité du mot «révolution" lors de son utilisation dans des contextes hétérogènes. Le cheminement de l'histoire de France entre la SaintBarthélémy et la Révolution de 1789 n'illustre donc pas le processus dialectique qui conduit à l'actualisation de l'esprit au sens hégélien, mais plutôt l'éternel retour de la volonté de puissance qui se dissimule sous l'apparence du progrès.

D'autre part le moment décisif que représente le 11 novembre 1642 pour l'avenir du peuple français n'est pas chargé de la signification messianique que prêtent Nietzsche et Benjamin ${ }^{13}$ à l'instant historique. En effet, l'irruption de Turlupin dans l'histoire ne correspond pas à une prise de conscience des virtualités illimitées de l'instant, pas plus qu'elle ne signifie une rupture radicale avec le passé. Elle pérennise au contraire l'ordre établi, garant de la monarchie absolue, qui présidera au destin de la France pendant encore un siècle et demi.

Pas plus que l'histoire témoigne du progrès de l'humanité, elle n'est régie par les lois de la causalité. Dans Turlupinl'enchaînement des événements échappe à toute logique préétablie selon laquelle le présent résulterait du passé et serait une transition vers l'avenir par une relation de cause à effet. L'acte « héroïque » du barbier n'est pas la conséquence logique des événements qui l'ont précédé, pas plus qu'il n'illustre la continuité du sens et sa circulation d'une époque à l'autre. Comme nous l'avons vu, c'est un quiproquo, non sa lucidité, qui a conduit Turlupin dans l'église des Trinitaires où son destin a basculé. Par ailleurs, ce n'est pas une conscience politique ancrée dans son époque qui déclenche le processus historique: tandis que l'aristocrate SaintChéron choisit le camp de la rébellion, le roturier Turlupin devient l'artisan de la contre-révolution. Si le perruquier ne trahissait pas lui aussi la cause de sa classe, le combat de son adversaire pourrait être interprété comme allant dans le sens de l'histoire. Or le chassé-croisé annule cette lecture. L'inversion des rôles va à l'encontre de toute logique de l'histoire, d'autant plus que l'aveuglement préside à l'affrontement des deux camps :

Ils se reconnurent. Et pourtant, ils ne se reconnurent pas. Le vicomte de Saint-Chéron voyait le perruquier Turlupin qui lui rasait la barbe toutes les semaines, sans se douter qu'il avait en face de lui la noblesse d'épée de France [...]. Turlupin, lui, voyait le commis d'un marchand de tissu de la rue des Apôtres, monsieur Gaspard [...] et il ne savait pas qu'avec l'homme dont son poignard frappait la poitrine s'effondrait aussi la révolution qui ne se relèverait que cent cinquante ans plus tard. (p. 178, Ed. all. pp. 147-148, souligné par nous, J.-P. C.)

31 Le sort d'une nation est ici entre les mains d'acteurs qui n'identifient pas l'adversaire et ignorent les tenants et les aboutissants du combat. Non seulement l'acte " héroïque " de Turlupin n'est que la conséquence d'une méprise, mais il est en réalité dicté par la peur d'être reconnu par monsieur Gaspard qui pourrait faire échouer son projet personnel. La déconstruction de la notion d'« héroïsme » s'opère à un triple niveau : la non-identification de l'adversaire, la dichotomie du voir et du savoir ainsi que l'immobilisation de l'histoire ôtent tout pathosà cette scène et font basculer l'action de Turlupin dans le grotesque. Comme le remarque monsieur Coquereau en conclusion : 
Dieu, à l'instar des grands seigneurs, s'est peut-être offert une bonne journée aux dépens d'un simple d'esprit (p. 184, Ed. all. p. 153). L'assimilation du " héros » à un histrion fait écho au commentaire introductif du narrateur à la fin du chapitre un. L'épisode d'histoire qui vient de nous être conté se résume donc à la "victoire » d'un bouffon sur le titan Richelieu.Les événements échappent à ceux qui sont censés les maîtriser. Ils deviennent le jouet d'un destinqui n'est rien d'autre qu'un mot creux plaqué sur l'absurde pour masquer le non-sens de l'histoire :

Le destin en décida autrement. Le destin suivait son propre chemin. La vieille France, vouée à la mort, devait une fois de plus triompher

des idées d'un temps nouveau. Le monde n'allait pas être frustré de l'éclat du règne du Roi-Soleil.

(p. 16, Ed. all. p. 12)

La définition du destin présupposée par cette assertion bat en brèche les notions métaphysiques de nécessité et de finalité pour s'annuler elle-même : le destin invoqué ici par le narrateur n'obéit pas à une logique qui orienterait le cours des événements. Si l'évolution historique condamne l'Ancien Régime au profit de la modernité de la pensée révolutionnaire, le destin œuvre à contre-courant. De la même façon, la victoire de la monarchie absolutiste de droit divin sur les idées progressistes est un anachronisme dans l'histoire mondiale(p.13, Ed. all. p.10). Aussi le vocable de destin relève ici d'une imposture de langage. Celle-ci dissimule que l'histoire n'obéit à aucune loi, mais qu'elle est livrée à un hasard aveugle. Dans cette même perspective, la Révolution française aurait pu avoir lieu 147 ans plus tôt, comme elle aurait pu ne pas avoir lieu du tout. Le règne de Louis xIV n'est qu'un accident de l'histoire quoi qu'en pensent ceux qui le considèrent comme l'aboutissement et l'apogée du système monarchique français.

Si la contingence préside au devenir du monde, quels enseignements peut-on tirer de l'histoire? De toute évidence, la date-clé du 11 novembre n'a pu qu'induire des rapprochements avec le 11 novembre 1918 dans les esprits des contemporains de Perutz. On sait que le romancier lui-même fut séduit un bref instant par l'expérience social-démocrate qui marqua les premières années de la République autrichienne. Or, lorsque Perutz écrit Turlupinen 1923, la social-démocratie est écartée du pouvoir depuis trois ans et la crise économique, qui secoue l'Autriche, n'est pas encore maîtrisée par Seipel. L'échec de la révolution de 1918 ne serait-il donc qu'une répétition de la révolution avortée de 1642 ? Le ton grotesque du roman et le libre jeu avec les données de l'histoire incitent le lecteur à la prudence et le dissuadent de pousser trop loin la comparaison. Néanmoins le choix de la date n'est pas innocent et la stratégie déconstructioniste du roman prend également pour cible l'histoire récente par le biais du parallèle suggéré. Cependant, contrairement à ses prédécesseurs ou à ses contemporains, Perutz ne présente pas l'étude du passé comme un moyen d'orientation dans le présent. Comme nous venons de le voir, l'histoire n'a, selon lui, aucun message à délivrer. Pas plus que le présent ne se nourrit du passé, il ne constitue, par rapport à lui, une étape signifiante vers un avenir meilleur. La mise en perspective de l'histoire par la référence aux idéaux républicains de 1789 et le triple niveau de temporalité du roman (1642/1789/1918) font apparaitre le scepticisme de l'auteur quant à la perfectibilité des institutions humaines.

L'originalité et l'attrait de la pensée sceptique à l'œuvre dans Turlupinréside dans le ton de la farce. Celle-ci utilise le rire comme arme contre les certitudes et le dogmatisme qui président à l'analyse des faits historiques. La démarche déconstructioniste de 
Perutz prend trois aspects de l'activité herméneutique pour cible : l'étude des sources, le repérage des idéologies et des enjeux politiques, la philosophie de l'histoire. La juxtaposition de sources diverses illustre davantage la subjectivité des acteurs de l'histoire que la fiabilité et la teneur en informations de leurs témoignages. Ce constat aboutit à un resserrement du texte sur l'anecdote qui est censée révéler le sens caché d'une énigme historique. Or, l'intrigue du roman met en évidence les intérêts personnels qui priment sur les enjeux politiques et président à la marche aveugle de l'histoire vers un avenir indéterminé. Aussi, toutes les interprétations proposées par la philosophie de l'histoire deviennent caduques dans la mesure où l'évolution historique n'est régie par aucune loi, mais présente les signes caractéristiques d'une bouffonnerie.

La stratégie narrative de Perutz prend les allures d'une farce qui consiste à différer la révélation du sens, pour finalement nier la présence de celui-ci. Ainsi notre auteur s'insurge par le rire contre l'herméneutique totalisante en quête de sens ultime. Il plaide, par ailleurs, pour une lecture qui met au jour le contexte, c'est à dire les coulisses ou la périphérie de l'histoire, ainsi que les signifiés hétérogènes qui se dissimulent sous l'apparente transparence des faits et de leur interprétation.

\section{NOTES}

1. Il parut tout d'abord simultanément dans la Vossische Zeitung à Berlin et dans l'édition du soir de la Neue Freie Presse à Vienne de décembre 1923 à février 1924. Il fut ensuite publié en 1924 aux Editions Langen à Munich.

2. Cf. H. Aust, Der historische Roman, Metzler, Stuttgart, 1994, p. 65.

3. Cf. A. Döblin, Der historische Roman und wir (1936) 7, in Ausgewählte Werke in Einzelbänden, Bd 26, Walter Verlag, Ölten, 1989, p. 299.

4. Cf. G. Genette, Figures III, Seuil, Paris, 1972, pp. 105-115. La diégèse est l'univers spatiotemporel du récit.

5. Cf. Genette, pp.105-115. Une prolepse est une anticipation du récit, l'annonce d'un fait à venir.

6. Ibid., pp. 90-105. L'analepse est l'évocation rétrospective d'un événement antérieur au présent du discours.

7. Cf. Jacques Derrida, La différence in Marges de la philosophie, Minuit, Paris, 1972, pp. 3-29. Le sens n'est jamais présent dans le signe. Celui-ci renvoie sans cesse aux significations antérieures et postérieures à son occurrence.

8. Nous nous référons dans cet article aux éditions suivantes: L. Perutz, Turlupin, Rowohlt, Hamburg, 1988. Traduction française de J.-C. Capèle, Le Livre de Poche, Biblio, Paris 1995. Trad. p. 8, Ed. all. p. 6.

9. Cf. C. Bremond, Logique du récit, Seuil, Paris, 1973.

10. Cf. G. Lukács, Der historische Roman, Probleme des Realismus, III, Luchterhand, 1965 et Balzac et le réalisme français, Maspero, Paris, 1969. Lukács définit le héros réaliste comme un type, c'est-à-dire un résumé d'histoire qui réunit en lui les caractéristiques de son époque et incarne les aspirations de la classe moyenne. 
11. Cf. A.-J. Greimas, Sémantique structurale, Larousse, 1966 et Du sens. Essais sémiotiques, Seuil, 1970.

12. Cf. L. Dällenbach, Le Récit spéculaire. Essai sur la mise en abyme, Seuil, Paris, 1977.

13. Cf. F. Nietzsche, Considérations inactuelles II, De l'utilité et des inconvénients de l'histoire, Gallimard, Coll. Folio/Essais, pp.93-169. W. Benjamin, Über den Begriff der Geschichte in Illuminationen, Ausgewählte Schriften, Suhrkamp, Frankfurt/Main, 1977.

Pour les deux philosophes, l'instant peut se muer en éternité. Pour qui sait déceler l'être qui se manifeste en lui. Ceci implique pour Nietzsche une attitude non-historique, c'est-à-dire l'art et la faculté d'oublier et de s'enfermer dans un horizon limité, ainsi qu'une démarche supra-historique par laquelle les sujets détournent le regard du devenir et le portent vers ce qui donne à l'existence un caractère d'éternité et de stabilité (p. 166).

Pour Benjamin, on peut déceler dans l'instant le signe d'un arrêt messianique du devenir, d'une révolution potentielle dans la lutte pour le passé opprimé, (p. 260).

\section{RÉSUMÉS}

La modernité du roman Turlupin de Leo Perutz réside dans le traitement irrévérencieux et la mise en énigme de l'histoire. Sur le mode ludique, l'auteur déconstruit les systèmes interprétatifs qui prétendent fixer le sens de l'évolution historique.

La stratégie narrative démonte les mécanismes de l'enquête historiographique : l'accumulation des sources dissimule paradoxalement plus qu'elle ne révèle. Les faits annoncés ne s'étant pas produits, la fiction romanesque relaye l'histoire et apporte la lumière sur l'avortement inexpliqué de la Révolution française de 1642.

Le bouffon Turlupin traverse les événements sans comprendre les enjeux du conflit dans lequel il intervient par mégarde: ce sont les intérêts personnels, la confusion et l'aveuglement qui président à la marche de l'histoire.

Perutz ne considère pas cette dernière comme une totalité homogène de signifiés. Il fait œuvre de déconstruction en prenant le contre-pied de l'historicisme et en opposant aux notions de progrès et de causalité celles de stagnation et de hasard. La mise en perspective de l'histoire fait apparaître l'éternel retour de l'identique. C'est une série de malentendus qui propulsent Turlupin au cœur de l'action et lui font inverser le cours des événements. Ceci réfute la théorie finaliste du devenir. Par ailleurs, l'intrigue du roman dément l'interprétation métaphysique qui fait du destin le moteur de l'évolution historique. Elle désigne l'absurde comme maître souverain du jeu et tourne en dérision tout décodage du sens de l'histoire.

Die Modernität des Romans Tur lupinvon Leo Perutz liegt in der respektlosen Behandlung der Geschichte, die er als undurchschaubares Rätsel vorführt. Auf spielerische Weise dekonstruiert der Verfasser die Deutungssysteme, die den Sinn der geschichtlichen Entwicklung festzulegen meinen.

Die Erzählstrategiezerlegt die Mechanismen der historiographischen Untersuchung: die angehäuften Quellen verschleiern paradoxerweise mehr, als sie enthüllen. Da die angekündigten Ereignisse ausbleiben, löst die Romanfiktion die Geschichte ab und bringt Licht ins unerklärte Scheitern der französischen Revolution von 1642. 
Der Narr Turlupin steht im Mittelpunkt des Geschehens, er versteht aber nicht, worauf der Konflikt hinauslauft, in den er aus Versehen eingreift: Eigennutz, Verwirrung und Verblendung bestimmen den Lauf der Geschichte.

Perutz betrachtet letztere nicht als eine homogene sinnstiftende Ganzheit. Er betreibt Dekonstruktion, indem er den Historismus widerlegt und an die Stelle von Fortschritt und Kausalität Stillstand und Zufall setzt. Die perspektivische Darstellung der Geschichte bringt die ewige Wiederkehr des Gleichen zum Vorschein. Infolge einer Reihe von Mißverständnissen wird Turlupin ins Zentrum der Ereignisse gestoßen, von wo aus er den Handlungsverlauf in die diametral entgegengesetzte Richtung umlenkt. So wird die teleologische Auffassung von der Entwicklung widerlegt. Außerdem straft die Romanhandlung die metaphysische Deutung Lüge, nach der das Schicksal den geschichtlichen Prozeß vorantreibt. Sie verweist auf das Absurde als den souveränen Spielmeister. Damit wird jedwede Auslegung der Geschichte zur Lächerlichkeit.

\section{AUTEUR}

\section{JEAN-PIERRE CHASSAGNE}

Université de Grenoble III 